\title{
EXPONENTIAL DECAY IN THE FREQUENCY OF ANALYTIC RANKS OF AUTOMORPHIC $L$-FUNCTIONS
}

\author{
P. MICHEL AND D. R. HEATH-BROWN
}

This note should be seen as a appendum to the work of E. Kowalski and the second author [KM1 and deals with the problem of bounding unconditionnally the order of vanishing at the critical point in a family of $L$ functions. This problem is illustrated in the particular case of $L$ functions of weight 2 primitive modular forms of prime level. Recall the notations from [KM1]: for $q$ a prime number, let $S_{2}(q)^{*}$ be the set of primitive forms of weight 2 and level $q$, normalized so that their first fourier coefficient is 1 ; for

$$
f(z)=\sum_{n \geqslant 1} \lambda_{f}(n) n^{1 / 2} \mathrm{e}(n z) \in S_{2}(q)^{*}
$$

let

$$
L(f, s):=\sum_{n \geqslant 1} \frac{\lambda_{f}(n)}{n^{s}}
$$

the associated (normalized) $L$-function; it admits analytic continuation to $\mathbf{C}$ with a functionnal equation relating $L(f, s)$ to $L(f, 1-s)$ and we call $r_{f}:=\operatorname{ord}_{s=1 / 2} L(f, s)$ the analytic rank of $f$. In KM1 the following was proved: there exists an absolute constant $C_{1} \geqslant 0$ such that for all q prime

$$
\sum_{f \in S_{2}(q)^{*}} r_{f} \leqslant C_{1}\left|S_{2}(q)^{*}\right| .
$$

After that much progress has been made concerning this question. In particular, in [KMV], a sharp explicit value was given for the constant $C_{1}\left(C_{1}=1.1891\right.$ for $q$ large enough). In the course of the proof, an uniform bound for the square of the ranks was obtained:

$$
\sum_{f \in S_{2}(q)^{*}} r_{f}^{2} \leqslant C_{2}\left|S_{2}(q)^{*}\right| .
$$

However, the latter improvment used only a slight variant of the methods of KM1. In fact, it is possible to pursue this idea further and it turns out that much more is true; this is the subject of the present note.

Theorem 0.1. There exist absolute constants $A, B>0$ such that, for all $q$ prime

$$
\sum_{f \in S_{2}(q)^{*}} e^{A r_{f}} \leqslant B\left|S_{2}(q)^{*}\right|
$$

Remark. It is possible to prove a bit more than this: namely one may replace $r_{f}$ by the number of zeros (counted with their multiplicity) in a small circle of radius $\sim 1 / \log q$ centred at $1 / 2$.

As a corollary we can look at the proportion of forms $f$ with analytic rank larger than a given $r$. This proportion decays exponentially with $r$ : 
Corollary 0.2. There exist absolute constants $A, B>0$ such that for all $r \geqslant 0$,

$$
\frac{\left|\left\{f \in S_{2}(q)^{*}, r_{f} \geqslant r\right\}\right|}{\left|S_{2}(q)^{*}\right|} \leqslant B e^{-A r}
$$

With more work, the above constants $A$ and $B$ could be computed explicitly and optimized (see for example [KM2] for the basic material necessary to do this). We may also remark that these results are very similar to the ones obtained by the first author and Fouvry concerning the distribution of the (arithmetic) rank in explicit families of twists of elliptic curves ([HB1, HB2, F] $)$.

Surprisingly, Theorem 0.1 is based on a very general principle and one can also obtain similar exponential upper bounds for the analytic rank for a wide class of families of $L$ functions, including the most basic example of the Dirichlet character $L$-functions. By refining slightly the work of Soundararajan [So], one can also treat the family of $L$-functions attached to quadratic characters. The general principle is that, as soon as one is able to control the mollified square of a family of $L$-functions, slightly to the right of the critical line, one obtains an exponential bound for the average of the analytic ranks.

As there is no real loss and no greater difficulty in axiomatizing this principle, we will do so, and deduce Theorem 0.1 as a special case.

Consider a finite probability space $(\Omega, \mu)$, where $\mu(\omega)>0$ for every $\omega \in \Omega$. For each $\omega \in \Omega$, suppose given a function $h_{\omega}(s)$ which is holomorphic in the half plane $\operatorname{Re}(s) \geqslant 0$. Moreover assume the following hypothesis on the variance of the function $h_{\omega}(s)-1$ :

Hypothesis 0.3. For some $B, C>0, M>2$, we have the bound

$$
\sum_{\omega \in \Omega}\left|h_{\omega}(\sigma+i t)-1\right|^{2} \mu(\omega) \leqslant C(1+|t|)^{B} M^{-\sigma}
$$

uniformly for $\sigma \geqslant(2 \log M)^{-1}$.

Note that in view of this hypothesis and the fact that $\mu(\omega)>0$, we have

$$
h_{\omega}(s)=1+O_{\omega}\left((1+|t|)^{B / 2} M^{-\sigma / 2}\right)
$$

for each $h_{\omega}$. Thus $h_{\omega}(s)$ is non-vanishing for sufficiently large $\sigma$. For any $\alpha \geqslant 0$ and $t_{1}<t_{2} \in \mathbf{R}$, we may therefore define $N\left(\omega, \alpha, t_{1}, t_{2}\right)$ to be the number of zeros $\rho$ of $h_{\omega}(s)$ such that

$$
\operatorname{Re}(\rho) \geqslant \alpha, t_{1} \leqslant \operatorname{Im}(\rho) \leqslant t_{2} .
$$

Clearly $N\left(\omega, \alpha, t_{1}, t_{2}\right)$ is finite. Our general result gives an upper bound for the $2 k$-th power of $N\left(\omega, \alpha, t_{1}, t_{2}\right)$ on average:

Theorem 0.4. With the above notations, assume that Hypothesis 0.3 is satisfied. Then for all $k \geqslant 1$, for all $\alpha \geqslant(\log M)^{-1}$, and all $t_{1}<t_{2}$, we have

$\sum_{\omega \in \Omega} N\left(\omega, \alpha, t_{1}, t_{2}\right)^{2 k} \mu(\omega) \ll C(k !)^{2}\left(48 \frac{k}{\alpha \log M}\right)^{2 k}\left(1+|t|+\frac{16 k}{\log M}\right)^{B} M^{-\alpha / 2}\left(1+\left(t_{2}-t_{1}\right) \log M\right)$

where we have set $|t|:=\operatorname{Max}\left(\left|t_{1}\right|,\left|t_{2}\right|\right)$. The constant involved in the Vinogradov symbol is absolute.

Remark. We have not tried to optimize the various explicit constants present in this bound, nor the exponent $1 / 2$ in $M^{-\alpha / 2}$.

In the next section, we prove this general result. Then, in the following one, we explain how Theorem 0.1 can be deduced from it. The necessary Hypothesis 0.3 in our setting will be provided by Proposition 4 of [KM1]. 
EXPONENTIAL DECAY IN THE FREQUENCY OF ANALYTIC RANKS OF AUTOMORPHIC L-FUNCTIONS

Acknowledgment. We would like to thank E. Kowalski and P. Sarnak for encouraging us to publish this note. This work began while the second author (P. M.) visited the Oxford Mathematical Institute. He wishes to thank the Institute for its hospitality and support. He is also supported by a membership at the Institut Universitaire de France.

\section{Proof of Theorem 0.4}

Obviously, we may assume $t_{2}-t_{1}=1 / \log M$. We start as in [KM1] by recalling the following lemma of Selberg $[\mathrm{S}]$ :

Lemma 1.1. (Selberg, [S, Lemma 14]). Let $h$ be a function holomorphic in the region

$$
\left\{s \in \mathbf{C} \mid \operatorname{Re}(s) \geqslant \alpha^{\prime}, t_{1}^{\prime} \leqslant \operatorname{Im}(s) \leqslant t_{2}^{\prime}\right\}
$$

and satisfying

$$
h(s)=1+o\left(\exp \left(-\frac{\pi}{t_{2}^{\prime}-t_{1}^{\prime}} \operatorname{Re}(s)\right)\right.
$$

in this region, uniformly as $\operatorname{Re}(s) \rightarrow+\infty$. Denoting the zeros of $f$ in the interior of the region by $\rho=\beta+i \gamma$, we have

$$
\begin{gathered}
2\left(t_{2}^{\prime}-t_{1}^{\prime}\right) \sum_{\rho} \sin \left(\pi \frac{\gamma-t_{1}^{\prime}}{t_{2}^{\prime}-t_{1}^{\prime}}\right) \sinh \left(\pi \frac{\beta-\alpha^{\prime}}{t_{2}^{\prime}-t_{1}^{\prime}}\right)=\int_{t_{1}^{\prime}}^{t_{2}^{\prime}} \sin \left(\pi \frac{t-t_{1}^{\prime}}{t_{2}^{\prime}-t_{1}^{\prime}}\right) \log \left|h\left(\alpha^{\prime}+i t\right)\right| d t \\
+\int_{\alpha^{\prime}}^{+\infty} \sinh \left(\pi \frac{\sigma-\alpha^{\prime}}{t_{2}^{\prime}-t_{1}^{\prime}}\right)\left(\log \left|h\left(\sigma+i t_{1}^{\prime}\right)\right|+\log \left|h\left(\sigma+i t_{2}^{\prime}\right)\right|\right) d \sigma
\end{gathered}
$$

(where the zeros are counted according to multiplicity).

We apply this lemma to each $h_{\omega}(s)$. We take $\alpha^{\prime}=\alpha / 2$, and $t_{2}^{\prime}=t_{2}+(\Delta-1) / 2 \log M$, $t_{1}^{\prime}=t_{1}-(\Delta-1) / 2 \log M$, where $\Delta=16 k$. Thus $t_{2}^{\prime}-t_{1}^{\prime}=\Delta / \log M$. Note that $\Delta>2 \pi$, so that the condition of Lemma 1.1 is satisfied, by (0.1). It therefore follows by positivity that

$$
\begin{gathered}
\frac{\alpha \log M}{\Delta}\left(t_{2}^{\prime}-t_{1}^{\prime}\right) N\left(\omega, \alpha, t_{1}, t_{2}\right) \leqslant 2\left(t_{2}^{\prime}-t_{1}^{\prime}\right) N\left(\omega, \alpha, t_{1}, t_{2}\right) \sin \left(\frac{\pi(\Delta-1)}{2 \Delta}\right) \sinh \left(\frac{\pi \alpha \log M}{2 \Delta}\right) \\
\leqslant \int_{t_{1}^{\prime}}^{t_{2}^{\prime}} \sin \left(\pi \frac{t-t_{1}^{\prime}}{t_{2}^{\prime}-t_{1}^{\prime}}\right) \log |h(\alpha / 2+i t)| d t \\
+\int_{\alpha / 2}^{+\infty} \sinh \left(\pi \frac{\sigma-\alpha / 2}{t_{2}^{\prime}-t_{1}^{\prime}}\right)\left[\log \left|h\left(\sigma+i t_{1}^{\prime}\right)\right|+\log \left|h\left(\sigma+i t_{2}^{\prime}\right)\right|\right] d \sigma .
\end{gathered}
$$

The reader should observe that the function $\log |h(s)|$ might take large negative values. Since we wish to apply Hölder's inequality, we first eliminate these large values by using the inequality $\log |1+x| \leqslant \log (1+|x|)$. This yields

$$
\begin{gathered}
\frac{\alpha \log M}{\Delta}\left(t_{2}^{\prime}-t_{1}^{\prime}\right) N\left(\omega, \alpha, t_{1}, t_{2}\right) \leqslant \int_{t_{1}^{\prime}}^{t_{2}^{\prime}} \log (1+|h(\alpha / 2+i t)-1|) d t \\
+\int_{\alpha / 2}^{+\infty} \exp \left(\pi \frac{\sigma-\alpha / 2}{t_{2}^{\prime}-t_{1}^{\prime}}\right)\left[\log \left(1+\left|h\left(\sigma+i t_{1}^{\prime}\right)-1\right|\right)+\log \left(1+\left|h\left(\sigma+i t_{2}^{\prime}\right)-1\right|\right)\right] d \sigma \\
:=I_{0}(\omega)+I_{1}(\omega)+I_{2}(\omega),
\end{gathered}
$$


say. Next we take the $2 k$-th power of this inequality, getting

$$
\left(\frac{\alpha \log M}{\Delta}\left(t_{2}^{\prime}-t_{1}^{\prime}\right) N\left(\omega, \alpha, t_{1}, t_{2}\right)\right)^{2 k} \leqslant 3^{2 k-1}\left(I_{0}(\omega)^{2 k}+I_{1}(\omega)^{2 k}+I_{2}(\omega)^{2 k}\right) .
$$

We only treat $I_{1}(\omega)^{2 k}$, the other terms being similar. By Hölder's inequality we have

$$
\begin{aligned}
& I_{1}(\omega)^{2 k} \leqslant\left(\int_{\alpha / 2}^{+\infty} \exp \left(-\pi \frac{2 k}{2 k-1} \frac{\sigma-\alpha / 2}{t_{2}^{\prime}-t_{1}^{\prime}}\right) d \sigma\right)^{2 k-1} \\
\times & \int_{\alpha / 2}^{+\infty} \exp \left(4 k \pi \frac{\sigma-\alpha / 2}{t_{2}^{\prime}-t_{1}^{\prime}}\right) \log \left(1+\left|h\left(\sigma+i t_{1}^{\prime}\right)-1\right|\right)^{2 k} d \sigma .
\end{aligned}
$$

The first factor is bounded by $\left(t_{2}^{\prime}-t_{1}^{\prime}\right)^{2 k-1}$. For the second term we use the fact that

$$
\log (1+|x|)^{k} \leqslant k !|x|,
$$

which follows from the inequality $y^{k} \leqslant k !\left(e^{y}-1\right)$. This allows us to bound the second integral by

$$
(k !)^{2} \int_{\alpha / 2}^{+\infty} \exp \left(4 k \pi \frac{\sigma-\alpha / 2}{t_{2}^{\prime}-t_{1}^{\prime}}\right)\left|h\left(\sigma+i t_{1}^{\prime}\right)-1\right|^{2} d \sigma .
$$

Since $4 \pi / 16<1$ the above integral is convergent, by 0.1 .

Next, averaging over $\omega$ and using Hypothesis $0.3\left(\right.$ since $\left.\alpha / 2 \geqslant(2 \log M)^{-1}\right)$, we obtain that

$$
\sum_{\omega \in \Omega} I_{1}(\omega)^{2 k} \mu(\omega) \leqslant(k !)^{2}\left(t_{2}^{\prime}-t_{1}^{\prime}\right)^{2 k} \frac{C}{\Delta(1-\pi / 4)}\left(1+|t|+\frac{16 k}{\log M}\right)^{B} M^{-\alpha / 2}
$$

This finishes the bound for $\sum_{\omega \in \Omega} I_{1}(\omega)^{2 k} \mu(\omega)$. We may bound the terms involving $I_{0}(\omega)$ and $I_{2}(\omega)$ similarly, thereby completing the proof of Theorem 0.4 .

\section{Application to zeros of $L$-Functions}

Next we turn to the proof of Theorem 0.1, in which we combine the preceding result with the method of explicit formulae of Riemann-Weil-Mestre KM1. Fix a smooth, even, non-negative test function $\phi$, compactly supported in $[-1,1]$, such that

$$
\operatorname{Re}(\hat{\phi}(s)) \geqslant 0, \text { for }|\operatorname{Re}(s)| \leqslant 1,
$$

where

$$
\hat{\phi}(s)=\int_{\mathbf{R}} \phi(x) e^{s x} d x
$$

denotes the Laplace transform of $\phi$. We shall assume that $\phi$ is normalized so that $\hat{\phi}(0)=1$. Let $\lambda>1 / 1000$ a parameter to be fixed later.

For $f \in S_{2}(q)^{*}$ we have (see [KM1]) the upper bound

$$
\begin{aligned}
\lambda r_{f} & \leqslant \phi(0) \log q-2 \sum_{p} \frac{\lambda_{f}(p)}{p^{1 / 2}} \phi\left(\frac{\log p}{\lambda}\right) \log p-2 \lambda \sum_{\operatorname{Re}\left(\rho-\frac{1}{2}\right) \geqslant \frac{1}{\lambda}} \operatorname{Re}\left\{\hat{\phi}\left(\lambda\left(\rho-\frac{1}{2}\right)\right)\right\}+O_{\phi}(\lambda) \\
(2.1) & \leqslant \phi(0) \log q-2 S(f)-2 \lambda \Xi(f)+O_{\phi}(\lambda),
\end{aligned}
$$


say, where $p$ runs over primes and $\rho$ run over the zeros of $L(f, s)$ of real part greater than $\frac{1}{2}+\frac{1}{\lambda}$. We aim to show that there is an absolute constant $C$ such that

$$
\sum_{f} r_{f}^{2 k} \leqslant(C k)^{2 k}\left|S_{2}(q)^{*}\right|
$$

for every integer $k \geqslant 1$. Then if the constant $A>0$ is chosen so that the series

$$
\sum_{k \geqslant 0} \frac{(A C k)^{2 k}}{(2 k) !}:=B
$$

is convergent, we will have

$$
\sum_{f} \exp \left(A r_{f}\right) \leqslant B\left|S_{2}(q)^{*}\right|
$$

as desired. By Stirling's formula we see that any $A<(e C)^{-1}$ is admissable.

We first make a trivial reduction. If one takes $\lambda=1$ and chooses $\phi$ appropriately in (2.1), one finds that

$$
r_{f} \leqslant 2 \log q
$$

for $q$ large enough. Thus in order to prove 2.2 we may suppose that $k \leqslant \log q$. We now take $\lambda$ to be of the form $\lambda=\theta \log q / k$, where $\theta>1 / 1000$ is an absolute constant to be determined later. It now remains to obtain bounds for

$$
\sum_{f} S(f)^{2 k} \text { and } \sum_{f} \Xi(f)^{2 k}
$$

2.1. The bound for $\sum_{f} S(f)^{2 k}$. In order to stay at an elementary level as much as possible, we will first consider the "harmonic" average, and prove the bound

$$
\sum_{f} \frac{1}{4 \pi(f, f)} S(f)^{2 k} \ll(C k \lambda)^{2 k}
$$

for some absolute $C$ and any integer $k \geqslant 1$. Here $(*, *)$ is the Petersson inner product on the space of weight 2 forms for $\Gamma_{0}(q)$. We may then reduce to the classical average by means of Cauchy-Schwarz

$$
\sum_{f} S(f)^{2 k} \leqslant\left(\sum_{f} 4 \pi(f, f)\right)^{1 / 2}\left(\sum_{f} \frac{1}{4 \pi(f, f)} S(f)^{4 k}\right)^{1 / 2} \ll(C k \lambda)^{2 k}\left|S_{2}(q)^{*}\right|
$$

using the bound

$$
\sum_{f} 4 \pi(f, f) \ll\left|S_{2}(q)^{*}\right|,
$$

for which see [M], for example. The advantage of using harmonic averaging is that we can apply Petersson's trace formula, which we state in the following simple form (see [KM1] for example).

Lemma 2.1. For $m \geqslant 1$ we have

$$
\sum_{f \in S_{2}(q)^{*}} \frac{1}{4 \pi(f, f)} \lambda_{f}(m)=\delta_{m, 1}+O\left(m^{1 / 2} q^{-3 / 2}\right) .
$$


Expanding $S(f)^{2 k}$, we see that all we need is to bound a linear combination of terms of the form

$$
\sum_{p_{1}} \sum_{p_{2}} \cdots \sum_{p_{j}} \sum_{f} \frac{1}{4 \pi(f, f)} \prod_{i=1}^{j}\left(\frac{\lambda_{f}\left(p_{i}\right) \log p_{i} \phi\left(\log p_{i} / \lambda\right)}{p_{i}^{1 / 2}}\right)^{\alpha_{i}}
$$

Here the $p_{i}$ run over distinct primes, and $\alpha_{1}, \alpha_{2}, \ldots, \alpha_{j}$ are fixed positive integers such that $\alpha_{1}+\cdots+\alpha_{j}=2 k$. In order to apply 2.5 we need to rewrite $\lambda_{f}(p)^{\alpha}$ in the form

$$
\lambda_{f}(p)^{\alpha}=a_{0, \alpha}+a_{1, \alpha} \lambda_{f}(p)+\cdots+a_{\alpha, \alpha} \lambda_{f}\left(p^{\alpha}\right),
$$

which may be done using the identity

$$
(2 \cos \theta)^{\alpha}=a_{0, \alpha}+a_{1, \alpha} 2 \cos \theta+\cdots+a_{\alpha, \alpha} 2 \cos \alpha \theta .
$$

Note in particular that the coefficients $a_{i, \alpha}$ are non-negative and that $a_{0, \alpha}=0$ if $\alpha$ is odd. Expanding the various $\lambda_{f}\left(p_{i}\right)^{\alpha_{i}}$ and applying (2.5), we see that we get a diagonal contribution (coming from the $\delta_{m, 1}$ term in (2.5)) if and only if all the $\alpha_{i}$ are even. This contribution is given by

$$
\sum_{p_{1}} \sum_{p_{2}} \cdots \sum_{p_{j}} \prod_{i=1}^{j} a_{0, \alpha_{i}}\left(\frac{\log p_{i} \phi\left(\log p_{i} / \lambda\right)}{p_{i}^{1 / 2}}\right)^{\alpha_{i}} \leqslant 2^{2 k} \sum_{p_{1}} \sum_{p_{2}} \cdots \sum_{p_{j}} \prod_{i=1}^{j}\left(\frac{\log p_{i} \phi\left(\log p_{i} / \lambda\right)}{p_{i}^{1 / 2}}\right)^{\alpha_{i}} .
$$

We may now recombine the various sums, to show that the diagonal contribution to the main sum $\sum_{f} \frac{1}{4 \pi(f, f)} S(f)^{2 k}$ is bounded by

$$
2^{2 k}\left(\sum_{p \leqslant e^{\lambda}} \frac{\phi(\log p / \lambda)^{2} \log ^{2} p}{p}\right)^{k} \leqslant(C k \lambda)^{2 k},
$$

for some constant $C$ depending only on our choice of $\phi$.

Similarly the total contribution coming from the error term in Petersson's formula is found to be

$$
\ll q^{-3 / 2}\left(\sum_{p \leqslant e^{\lambda}} \phi(\log p / \lambda) \log p\right)^{2 k} \ll C^{2 k} e^{2 k \lambda} q^{-3 / 2} \ll C^{2 k} q^{2 \theta-3 / 2} .
$$

Hence, as long as $\theta<3 / 4$, we may conclude that

$$
\sum_{f} \frac{1}{4 \pi(f, f)} S(f)^{2 k} \ll(C k \lambda)^{2 k},
$$

for some absolute constant $C$. This completes the proof of 2.3

Remark It is also possible to prove (2.3) directly by means of the trace formula for Hecke operators.

2.2. The bound for $\sum_{f} \Xi(f)^{2 k}$. We begin by noting that repeated integration by parts produces an estimate

$$
\hat{\phi}(s) \ll_{l} \frac{1}{(1+|\operatorname{Im}(s)|)^{l}} e^{\operatorname{Re}(s)}
$$

for any integer $l \geqslant 1$. If we split the strip $\left\{1 / \lambda \leqslant \operatorname{Re}\left(\rho-\frac{1}{2}\right) \leqslant 1\right\}$ into rectangles of width $1 / \lambda$, of the form

$$
\left\{1 / \lambda \leqslant \operatorname{Re}\left(\rho-\frac{1}{2}\right) \leqslant 1, \frac{m}{\lambda} \leqslant \operatorname{Im}(s)<\frac{m+1}{\lambda}\right\}
$$


and observe that (for $\beta \geqslant 1 / \lambda$ )

$$
e^{\lambda(\beta-1 / 2)} \ll \lambda \int_{\frac{1}{2 \lambda}}^{\beta-\frac{1}{2}} e^{\lambda \alpha} d \alpha
$$

we find that

$$
\Xi(f) \ll_{l} \lambda \sum_{m \geqslant 0} \frac{1}{(1+m)^{l}} I_{m}
$$

where

$$
I_{m}=\int_{\frac{1}{2 \lambda}}^{\frac{1}{2}} n\left(f, \alpha, \frac{m}{\lambda}, \frac{m+1}{\lambda}\right) e^{\lambda \alpha} d \alpha .
$$

Here we define $n\left(f, \alpha, t_{1}, t_{2}\right)$ to be the number of zeros of $L\left(f, \frac{1}{2}+s\right)$ in the region

$$
\left\{\operatorname{Re}(s) \geqslant \alpha, t_{1} \leqslant \operatorname{Im}(s) \leqslant t_{2}\right\}
$$

We apply Hölder's inequality to $I_{m}$, whence

$$
I_{m}^{2 k} \leqslant\left(\int_{\frac{1}{2 \lambda}}^{\frac{1}{2}} e^{-\lambda \alpha} d \alpha\right)^{2 k-1}\left(\int_{\frac{1}{2 \lambda}}^{\frac{1}{2}} n\left(f, \alpha, \frac{m}{\lambda}, \frac{m+1}{\lambda}\right)^{2 k} e^{(4 k-1) \lambda \alpha} d \alpha\right) \leqslant \lambda^{1-2 k} J(f, m, k),
$$

where

$$
J(f, m, k)=\int_{\frac{1}{2 \lambda}}^{\frac{1}{2}} n\left(f, \alpha, \frac{m}{\lambda}, \frac{m+1}{\lambda}\right)^{2 k} e^{4 k \lambda \alpha} d \alpha
$$

Thus

$$
\Xi(f)^{2 k} \leqslant C_{l}^{k} \lambda\left(\sum_{m \geqslant 0} \frac{1}{(1+m)^{2}}\right)^{2 k-1} \sum_{m \geqslant 0} \frac{1}{(1+m)^{2 k l-(4 k-2)}} J(f, m, k),
$$

for some $C_{l}$ depending only on $l$.

We are now ready to apply Theorem 0.4 . We shall take our space $\Omega$ to be the set $S_{2}(q)^{*}$, equipped with the uniform probability measure $\left(\mu(f)=1 /\left|S_{2}(q)^{*}\right|\right)$. The function $h_{f}(s)$ will have the form $h_{f}(s)=L\left(f, \frac{1}{2}+s\right) M\left(f, \frac{1}{2}+s\right)$ where $M(f, s)$ is a suitable 'mollifier'. Such functions were constructed in [KM1], so that Hypothesis 0.3 is satisfied for $M=q^{1 / 5}$ (say) and for some absolute constants $B, C$ (Proposition 4.). We observe that $n\left(f, \alpha, t_{1}, t_{2}\right) \leqslant$ $N\left(f, \alpha, t_{1}, t_{2}\right)$, in the notation of Theorem 0.4 . Thus, on recalling that

$$
k^{-1} \log q \ll \lambda \ll k^{-1} \log q,
$$

and that $M=q^{1 / 5}$, we deduce that

$$
\left|S_{2}(q)^{*}\right|^{-1} \sum_{f} J(f, m, k) \ll C^{k}(k !)^{2}(1+m)^{B} \int_{\frac{1}{2 \lambda}}^{\frac{1}{2}} q^{-\alpha / 10} e^{4 k \lambda \alpha} d \alpha,
$$

for some constant $C$. We may now choose $l=B+2$, say, and $\lambda=(40 k)^{-1} \log q$, to obtain

$$
\sum_{f} J(f, m, k) \ll C^{k}(k !)^{2} \lambda^{-1}\left|S_{2}(q)^{*}\right|
$$

for some absolute constant $C$. It then follows that

$$
\sum_{f} \Xi(f)^{2 k} \ll C^{k}(k !)^{2}\left|S_{2}(q)^{*}\right|
$$

with a new constant $C$. Finally, combining (2.4), 2.6) and (2.1), we obtain 2.2. 


\section{REFERENCES}

[F] E. Fouvry: Sur le comportement en moyenne du rang des courbes $y^{2}=x^{3}+k$. Séminaire de Théorie des Nombres, Paris, 1990-91, 61-84, Progr. Math., 108, Birkhäuser 1993.

[HB1] D.R. Heath-Brown: The size of Selmer groups for the congruent number problem. Invent. Math. 111 (1993), no. 1, 171-195.

[HB2] D.R. Heath-Brown: The size of Selmer groups for the congruent number problem. II. Invent. Math. 118 (1994), no. 2, 331-370.

[KM1] E. Kowalski and P. Michel : The analytic rank of $J_{0}(q)$ and zeros of automorphic L-functions, to appear in Duke Math. Journal.

[KM2] E. Kowalski and P. Michel: An explicit upper bound for the rank of $J_{0}(q)$, to appear in Israel Journal of Math.

[KMV] E. Kowalski, P. Michel and J. M. VanderKam: Non-vanishing of high derivatives of automorphic $L$-functions at the center of the critical strip, to appear in J. Reine Angew. Math.

[M] R. Murty: The analytic rang of $J_{0}(N)$, (K. Dilcher Edt.) CMS Conf. Proc. 15, 263-277 (1995).

[S] A. Selberg: Contributions to the theory of Dirichlet's L-functions, Skr. utg. av Det Norske VidenskapsAkademi, Mat. Nat. Kl. (1946) No 3, 1-62.

[So] K. Soundararajan: Non-vanishing of quadratic Dirichlet L-functions at $s=1 / 2$, preprint.

IUF et Université Montpellier II CC 051, 34095 Montpellier Cedex 05, FRANCE.

E-mail address: michel@darboux.math.univ-montp2.fr

Mathematical Institute, 24-29 St. Giles', Oxford, OX1 3LB, ENGLAND.

E-mail address: : rhb@maths.ox.ac.uk 